Reprod. Nutr. Dévelop., 1983, 23 (4), 679-692.

\title{
Réactions de précipitation entre les produits de sécrétion des oviductes chez les Amphibiens
}

P. JÉGO, A. CHESNEL, J. JOLY

Laboratoire de Biologie de la Reproduction, Groupe de Recherches en Biologie cellulaire, (C.N.R.S. LA 256)

Université de Rennes, 35042 Rennes Cedex, France.

Summary. Precipitin reactions between secretory products in the amphibian oviduct.

The existence of precipitin reactions between some molecules in egg jellies loviduct secretions) of tailed amphibians (Amphibia caudata) has been demonstrated by double diffusion on agarose plates. These reactions do not exist in frogs and toads (Amphibia salientia).

One precipitin reaction was related to compounds with a common molecular site of interaction for all $A$. caudata : all cross-species reactions were possible; a common antigenic site has been shown.

Another precipitin reaction, positively demonstrated in Pleurodeles waltl, probably exists in other $\boldsymbol{A}$. caudata.

The putative influence of these reactions on egg jelly-spermatozoon interactions has been discussed. An homology between these intra-egg jelly reactions and cortical granule content-egg jelly reactions in $A$. salientia has been suggested.

\section{Introduction.}

Chez tous les Amphibiens, les œufs sont recouverts d'une enveloppe gélatineuse élaborée par l'oviducte. Cette enveloppe, formée de plusieurs couches distinctes appelées gangues ovulaires, joue un rôle physiologique dans le déroulement normal de la fécondation (Bataillon, 1919 ; Good et Daniel, 1943 ; Kambara, 1953 ; voir revue de Metz, 1967). Des interactions entre les spermatozoïdes et les gangues ovulaires sont responsables de la préparation des spermatozoïdes qui vont féconder l'ovocyte et du blocage des spermatozoïdes surnuméraires.

L'étude directe des modifications des spermatozoïdes au contact des gangues ovulaires n'a pas permis jusqu'à présent de déterminer la nature réelle des interactions qui sont en cause. La préparation à la fécondation des spermatozoïdes d'Amphibiens, a été qualifiée de "capacitation " par Shivers et James (1970) par analogie avec ce qui se passe dans les voies génitales femelles des Mammifères, mais on ne connaît la nature des mécanismes moléculaires responsables de cette capacitation, ni dans un cas, ni dans l'autre (Shapiro et Eddy, 
1980). Pour Wyrick et al. (1974), et Grey et al. (1976), le blocage des spermatozoïdes surnuméraires est assuré par des mécanismes chimiques; mais Cross et Elinson (1980) invoquent des mécanismes électriques. Les analyses chimiques qui ont été effectuées ont montré combien les gangues ovulaires étaient hétérogènes et complexes, aussi bien chez les Anoures (Yurewicz et al., 1975), que chez les Urodèles (Jégo, 1974, 1976). Certains produits de sécrétion de l'oviducte de Pleurodeles waltl (gangues ovulaires non excrétées) sont capables de provoquer des réactions de précipitation de type " antigène-anticorps » (Jégo et al., 1976).

Dans le présent travail, où ces réactions de précipitation sont étudiées de manière plus approfondie chez le Pleurodèle, nous avons cherché à les mettre en évidence chez d'autres Amphibiens Urodèles et Anoures. Ces recherches ont porté sur les produits de sécrétion des différentes régions de l'oviducte avant leur excrétion d'une part et après leur excrétion (gangues ovulaires) d'autre part.

\section{Matériel et méthodes.}

1. - Animaux. - Pleurodeles walt/ Michah. et Ambystoma mexicanum sont élevés au laboratoire. Salamandra salamandra (Linné), Triturus helveticus (Linné) Triturus marmoratus (Latreille), Bufo bufo (Linné), Rana esculenta (Linné) et Alytes obstetricans (Laurenti) ont été capturés dans les environs de Rennes (France). Quelques exemplaires d'Hynobius nebulosus (Temminck et Schlegel) proviennent de l'élevage de M. R. Thorn (Luxembourg). Des exemplaires de Notophtalmus viridescens (Rafinesque) ont été achetés chez Xenopus Ltd (England).

2. - Prélèvement des différents segments d'oviducte. - Chez tous les Urodèles étudiés et chez Alytes obstetricans on distingue, à I'œil nu, plusieurs segments dans l'oviducte. Ces différents segments sont schématisés dans la figure 1. Dans une direction cranio-caudale, on observe tout d'abord, faisant suite à la trompe ciliée, un segment antérieur blanchâtre relativement transparent (A ou a dans le texte). Le segment moyen blanc-laiteux qui lui fait suite, est nettement plus opaque à la lumière $(\mathrm{M}$ ou $\mathrm{m})$; le reste de l'oviducte constitue le segment postérieur ( $P$ ou $p$ ). Ces segments sont soit prélevés en entier, soit découpés en plusieurs tronçons qui, dans ce cas, porteront dans le texte les indices $1,2,3 \ldots$ selon leur situation cranio-caudale.

Chez Bufo bufo et Rana esculenta on ne peut pas distinguer, à l'œil nu, différents segments dans l'oviducte. Dans ces deux cas, les oviductes ont été découpés en très nombreux morceaux repérés antéro-postérieurement et désignés par $0_{1}, \mathrm{O}_{2}, \ldots \mathrm{O}_{6}$.

3. - Préparation des extraits d'oviductes. - Après prélèvement, les segments sont homogénéisés grâce à un appareil de Potter dans du tampon tris- $\mathrm{HCl}$ $0,1 \mathrm{M}$ à pH 7 ou 8,2 . Les homogénats sont ensuite centrifugés à $20000 \mathrm{~g}$ pendant une heure et les expériences sont réalisées sur les surnageants ainsi obtenus, dénommés extraits d'oviductes.

\section{4. - Prélèvement des gangues ovulaires.}

a) Chez Pleurodeles walt/. Dans le but d'obtenir des extraits de référence les plus 


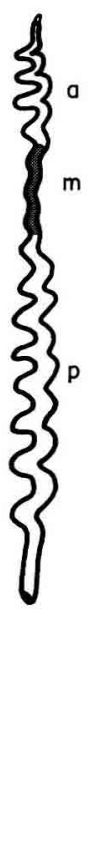

2

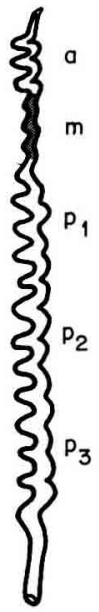

3

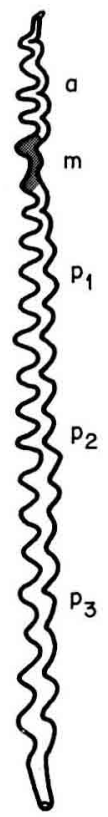

4
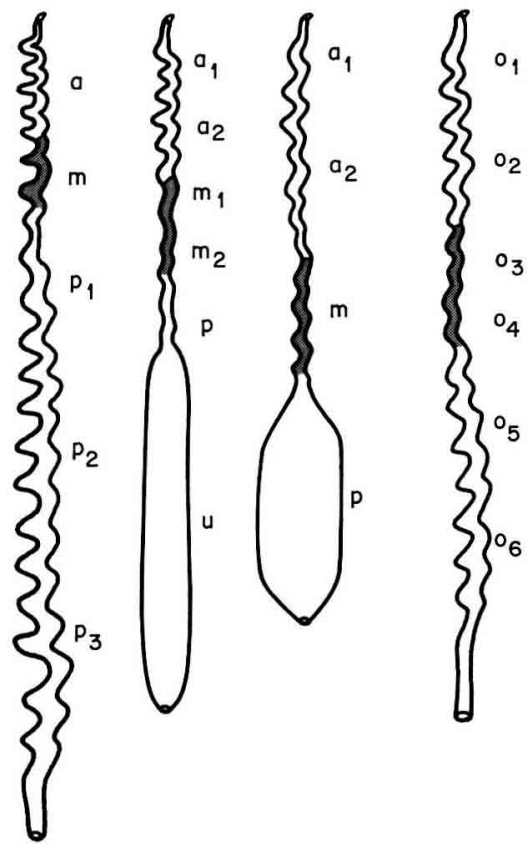

6

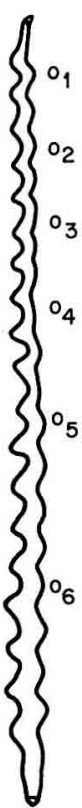

9

FIG. 1. - Schémas de l'oviducte des Amphibiens étudiés. 1 : Pleurodeles waltl ; 2 : Triturus helvetjcus ; 3 : Notophtalmus viridescens ; 4 : Triturus marmoratus ; 5 : Ambystoma mexicanum ; 6 : Salamandra salamandra; 7 : Hynobius nebulosus; 8 : Alytes obstetricans; 9 : Bufo bufo et Rana esculenta.

Dans l'oviducte de tous les Urodèles, on distingue de l'avant vers l'arrière : un segment antérieur (dénommé A chez le Pleurodèle et a chez les autres Urodèles) qui fait suite à la trompe ciliée : puis un segment moyen plus blanchâtre $(M, m)$ représenté en grisé sur les schémas ; un segment postérieur $(P, p)$ généralement très développé, sauf chez la salamandre (espèce à gestation utérine) et enfin un utérus peu glandulaire et très court, sauf chez la salamandre (u).

Chez les Anoures, on ne peut généralement pas distinguer à l'œil nu les différents segments qui ont été appelés $o_{1}, o_{2} \ldots o_{6}$ selon leur situation antéropostérieure ; sauf chez Alytes obstetricans où une région est plus blanchâtre que les autres $\left(o_{3}+o_{4}\right)$.

purs possible, notamment pour les expériences d'immunochimie, nous avons mis au point une technique de séparation des différentes gangues ovulaires de Pleurodeles. Cette technique qui a déjà été décrite (Jégo, 1976), peut se résumer ainsi : la partie la plus externe des gangues ovulaires est ôtée par dissection à la pince ; une exposition aux rayons ultra-violets $(\lambda=254 \mathrm{~nm} ; 30 \mathrm{~min})$ liquéfie les gangues externes restantes. Après de nombreux lavages, la gangue moyenne est déchirée ; l'ovocyte est éliminé ; la gangue moyenne qui est très résistante et la gangue interne sont séparées par centrifugations successives entrecoupées de lavages. En pratique, cette méthode n'est applicable qu'à des gangues ovulaires déposées dans l'eau depuis $12 \mathrm{~h}$ environ.

Certaines expériences ont porté sur des gangues ovulaires très fraîchement pondues; dans ce cas c'est un mélange de gangues interne et moyenne très 
sommairement débarrassé des ovocytes et des gangues externes et dénommé extrait brut de gangues fraîches, qui a été utilisé. Les ovocytes eux-mêmes ont été conservés après un rapide lavage.

b) Chez Salamandra salamandra et Hynobius nebulosus. Les extraits de gangues ovulaires de Salamandra ont été obtenus après séparation à la pince des gangues externe, moyenne et interne entourant de très jeunes embryons prélevés dans l'utérus de femelles gestantes.

Les gangues ovulaires d'Hynobius nebulosus se présentent différemment de celles des autres Urodèles. Sous un sac externe assez consistant, plusieurs gangues peuvent être séparées à la pince avant d'atteindre la gangue la plus interne.

Après prélèvement, les gangues sont homogénéisées puis lyophilisées, sauf lorsqu'il s'agit de gangues très fraîchement pondues qui sont utilisées sans aucun traitement supplémentaire.

5. - Préparation des immunsérums. - Deux catégories d'immunsérums de Lapin ont été utilisées : d'une part, un immunsérum dirigé contre la gangue ovulaire interne de Pleurodeles, dénommé " sérum $\sigma_{1}$ "; d'autre part, un immunsérum dirigé contre la gangue ovulaire moyenne de Pleurodeles, dénommé " sérum $\sigma_{2} n$.

Les immunisations ont été réalisées par injections sous-cutanées hebdomadaires de $150 \mu \mathrm{g}$ de gangue lyophilisée associée à un adjuvant de Freund complet puis incomplet (12 à 15 injections au total selon les lapins).

6. - Diffusion sur gel d'agarose. - Les extraits d'oviducte $14 \mathrm{mg}$ de protéines totales $/ \mathrm{ml}$ ) ou de gangues ( 2 à $8 \mathrm{mg}$ de protéines totales $/ \mathrm{ml}$ ), sont déposés dans les puits d'un gel d'agarose à $1 \%$ dans le tampon tris- $\mathrm{HCl} 0,1 \mathrm{M}$ (pH 8,2 ou 7) selon le protocole classique utilisé pour les immunodiffusions type Ouchterlony (1949). Les arcs se formant entre les extraits d'oviductes ou de gangues en absence de tout immunsérum, seront dénommés « arcs de précipitation ».

Dans certaines expériences, les propriétés de précipitation ont été comparées aux propriétés antigéniques après dépôt dans certains puits du gel d'agarose des sérums $\sigma_{1}$ ou $\sigma_{2}$; afin de ne pas créer de confusion, les arcs résultant d'interactions entre antigènes et anticorps, seront dénommés « arcs d'immunoprécipitation $)$.

Après incubation en chambre humide à $37{ }^{\circ} \mathrm{C}$ pendant 3 jours, les gels d'agarose sont lavés pendant une semaine par $\mathrm{NaCl} 9 \%$, séchés puis colorés au noir-amide.

\section{Résultats.}

\section{A. - Mise en évidence de réactions de précipitation entre certains produits de sécrétion de l'oviducte antérieur et moyen chez les Urodèles.}

Dans la planche I sont présentés quelques exemples montrant que les produits de sécrétion des oviductes antérieur et moyen de différents Urodèles, réagissent entre eux lorsqu'ils sont confrontés sur gel d'agarose en absence de tout anticorps exogène. 


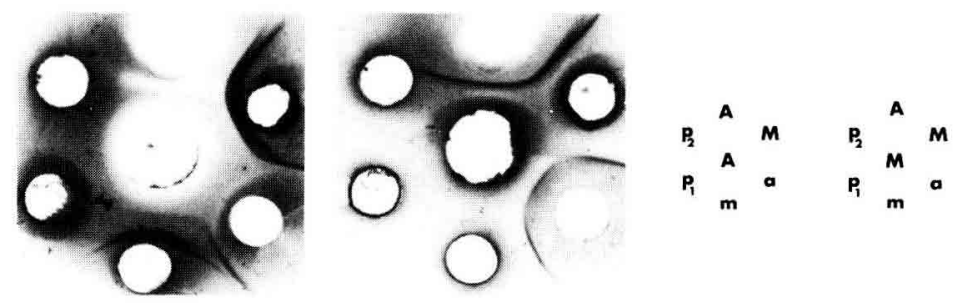

Notophtalmus viridescens et $P$. w.
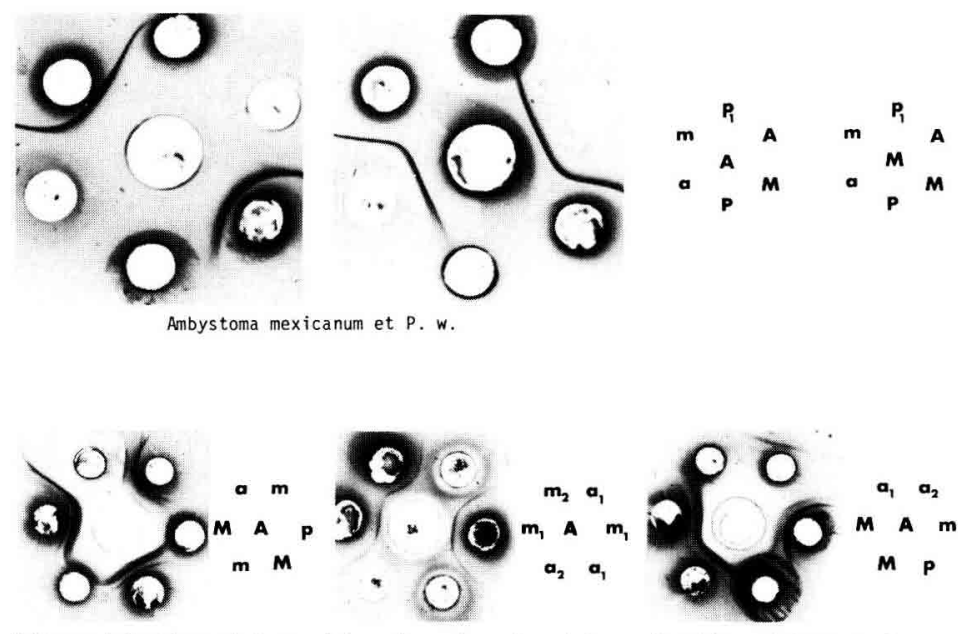

Triturus helveticus et $P$. W. Salamandra salamandra et $P$. W. Hynobius nebulosus et $P$. W

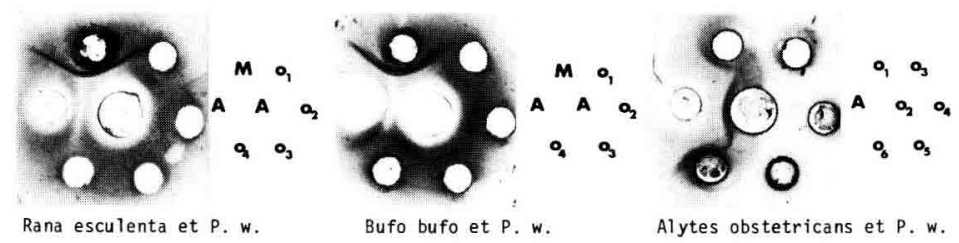

PLANCHE I

Réactions de précipitation sur gel d'agarose entre les produits de sécrétion de différents segments d'oviductes d'Amphibiens.

$A, M, P$ : segments antérieur, moyen et postérieur du Pleurodèle $(P . W$.$) .$

$a, m, p$ : segments antérieur, moyen et postérieur de l'Urodèle dont le nom est indiqué sous les clichés (1, 2 désignent respectivement les régions antérieure et postérieure du segment).

$\mathrm{o}_{1}, \mathrm{o}_{2} \ldots \mathrm{o}_{6}$ : portions de l'oviducte de l'Anoure dont le nom est indiqué sous le chiché (découpage antéro-postérieur\}.

Les segments antérieurs d'une part et moyens d'autre part des oviductes d'Urodèles, quels qu'ils soient, forment entre eux des arcs de précipitation en absence de tout anticorps exogène. Les arcs majeurs obtenus présentent des communautés totales montrant l'ubiquité des réactions correspondantes $\left(L_{1}-I_{1}\right)$ chez les Urodèles. Les arcs $L_{2}-I_{2}$ observés avec le Pleurodèle existent peut-être chez Salamandra, Hynobius et Triturus helveticus lon observe de nombreux arcs de précipitation mais leur communauté totale avec les arcs $\mathrm{L}_{2}-\mathrm{I}_{2}$ du Pleurodèle n'est pas sûre). Aucune des portions d'oviductes d'Anoures ne renferme les composés $L_{1}, L_{2}$ ou $l_{1}$; seuls des composés de type $I_{2}$ forment des arcs diffus contre l'oviducte antérieur du Pleurodèle. 
1. - Chez Pleurodeles waltl. - Chez Pleurodeles qui nous a servi de référence, on distingue nettement deux familles d'arcs de précipitation (fig. 2). A peu près à égale distance des deux puits de dépôt, on observe une première famille d'arcs très nets composée d'un arc épais se subdivisant en 4 arcs sur les côtés d'une part, et d'un arc moins épais parfois confondu avec le précédent $d^{\prime}$ autre part. Afin de faciliter l'exposé, nous appellerons respectivement $L_{1}$ et $I_{1}$ les produits de sécrétion de l'oviducte antérieur et de l'oviducte moyen, responsables de ces arcs. Très près du puits de dépôt de l'extrait d'oviducte moyen (parfois même en arrière), on observe une deuxième famille d'arcs beaucoup plus flous, composée d'un arc majeur et constant et de deux à quatre arcs plus discrets et inconstants. Les produits de sécrétion de l'oviducte antérieur et de l'oviducte moyen, responsables de ces arcs, seront appelés respectivement $L_{2}$ et $I_{2}$.

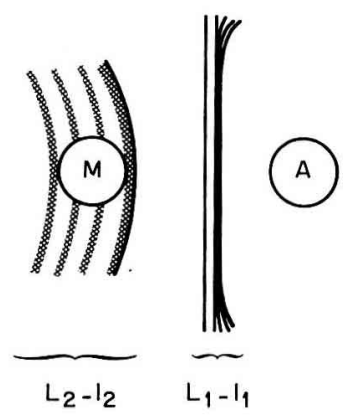

FIG. 2. - Schéma des arcs de précipitation obtenus chez le Pleurodèle. A : extrait d'oviducte Antérieur, $M$ : extrait d'oviducte Moyen.

On distingue une première famille d'arcs très nets composée d'un premier arc majeur se subdivisant en quatre arcs latéralement et $d^{\prime}$ un arc mineur parfois confondu avec le précédent; l'ensemble de ces deux arcs a été dénommé arcs $L_{1}-I_{1}$. Très près du puits de dépôt de l'extrait d'oviducte moyen, on distingue une deuxième famille d'arcs nettement plus flous, comnosée d'un arc majeur et de deux à quatre arcs inconstants ; l'ensemble de cette deuxième famille d'arcs a été dénommée $L_{2}-\left.\right|_{2}$.

2. - Chez les autres Urodèles étudiés. - Chez tous les Urodèles que nous avons étudiés, on retrouve des arcs de précipitation entre les produits de l'oviducte antérieur et de l'oviducte moyen lorsqu'ils sont confrontés sur agarose. Toutes les réactions croisées interspécifiques sont possibles; les arćs obtenus sont toujours en communauté totale avec les arcs $\mathrm{L}_{1}-\mathrm{l}_{1}$ de Pleurodeles, sauf peutêtre pour Hynobius nebulosus dont certains arcs sont en communauté totale mais d'autres seulement en communauté partielle avec les arcs obtenus chez Pleurodeles.

Des arcs apparus avec certains spécimens de Triturus helveticus, Triturus marmoratus, Salamandra salamandra ou Hynobius nebuilosus présentent des communautés partielles avec les arcs $\mathrm{L}_{2}-\mathrm{I}_{2}$.

Chez Notophtalmus viridescens et Ambystoma mexicanum, nous n'avons observé aucun indice susceptible de faire suspecter la présence des produits $L_{2}$. Les produits $\mathrm{I}_{2}$ existent dans tous les extraits étudiés. 
3. - Chez les Anoures étudiés. - Nous n'avons jamais pu mettre en évidence de réaction de type $L_{1}-I_{1}$ ou $L_{2}-t_{2}$ chez les Anoures que nous avons étudiés. Les différentes régions de l'oviducte, difficiles à distinguer à l'œil nu chez Rana esculenta et Bufo bufo, mais discernables chez Alytes obstetricans, ne semblent renfermer aucune molécule susceptible de réagir avec les produits $L_{1}, I_{1}$ ou $I_{2}$. Toutes les régions renferment par contre et de manière à peu près uniforme, des produits qui réagissent avec $L_{2}$ de Pleurodeles.

\section{B. - Etude immunochimique aes produits responsables des réactions de préci- pitation (Planche II).}

\section{1. - Chez Pleurodeles waltl.}

a) Avec le sérum $\sigma_{1}$. L'extrait d'oviducte antérieur forme quatre groupes d'arcs d'immunoprécipitation. En allant du puits de dépôt de l'extrait d'oviducte vers le puits de dépôt du sérum, on observe : un premier arc très léger que nous appellerons $x$; un groupe d'arcs intenses correspondant aux produits $L_{1}$, un arc très fin (très souvent masqué par les autres) que nous appellerons y et enfin un groupe d'arcs très diffus bien qu'intenses correspondant aux produits $L_{2}$.

L'extrait d'oviducte moyen forme plusieurs arcs ; I'un d'eux est en communauté totale avec l'arc $\mathrm{y}$.

b) Avec le sérum $\sigma_{2}$. L'extrait d'oviducte antérieur forme plusieurs arcs d'immunoprécipitation; deux d'entre eux sont en communauté totale respectivement avec $x$ et $y$.

L'extrait d'oviducte moyen forme plusieurs groupes d'arcs d'immunoprécipitation. Les uns, très intenses et proches du puits de dépôt de l'extrait d'oviducte, correspondent aux produits $I_{1}$. Parmi les autres arcs, l'un est en communauté totale avec $y$.

c) Avec le sérum témoin (lapin non immunisé $\left(\sigma_{0}\right)$. L'extrait d'oviducte antérieur forme un arc diffus qui est l'arc $x$ précédemment observé ; il s'agit d'un arc de précipitation de type $\mathrm{L}_{2}-\mathrm{I}_{2}$.

L'extrait d'oviducte moyen ne forme aucun arc contre le sérum de lapin non immunisé.

d) Communautés entre les arcs d'immunoprécipitation et les arcs de précipitation entre segments. Le résultat le plus net est l'absence apparente des arcs $L_{1}-I_{1}$ observée chaque fois que les extraits d'oviducte antérieur et moyen sont mis en présence du sérum $\sigma_{2}$. En réalité, ces arcs sont déplacés vers le puits de dépôt de l'extrait d'oviducte moyen et sont en communauté totale avec les arcs d'immunoprécipitation $I_{1}-\sigma_{2}$. Certains anticorps anti- $I_{1}$ reconnaissent donc sur $I_{1}$ les mêmes sites moléculaires que les produits $L_{1}$.

Les arcs $L_{2}-t_{2}$ présentent, pour certains d'entre eux, des communautés partielles (éperons) avec les arcs $L_{2}-\sigma_{1}$ et des communautés totales pour d'autres. Les arcs $L_{1}-l_{1}$ présentent des communautés partielles avec les arcs $L_{1}-\sigma_{1}$. Ainsi les sites moléculaires de $L_{1}$ et de $L_{2}$ impliqués respectivement dans les réactions $L_{1}-l_{1}$ et $\mathrm{L}_{2}-\mathrm{I}_{2}$ ne sont généralement pas reconnus par les anticorps des sérums $\sigma_{1}$ ou $\sigma_{2}$ qui reconnaissent par contre d'autres régions de ces molécules.

Les produits $\mathrm{I}_{2}$ ne sont reconnus par aucun sérum. 

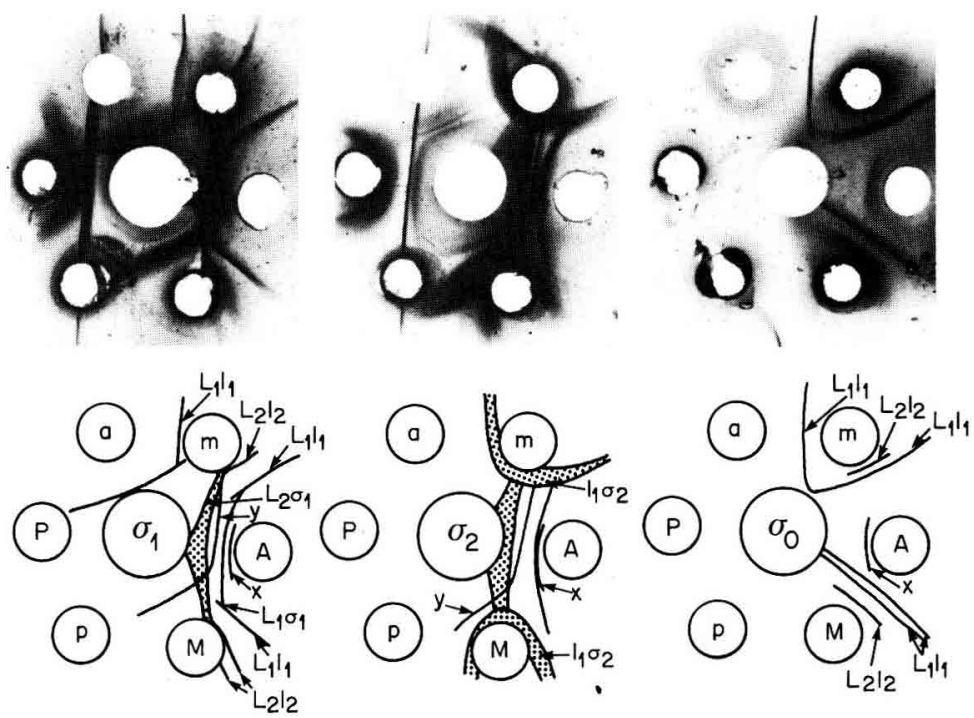

Anbystoma mexicanum et P.w.
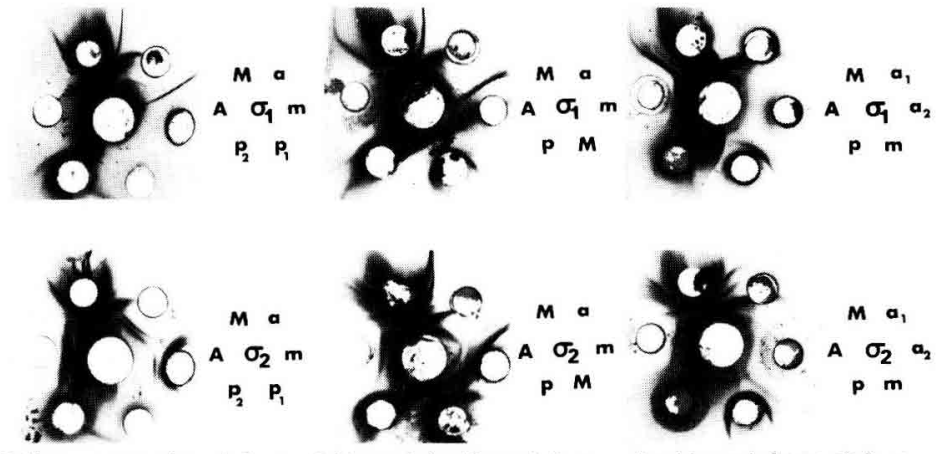

Triturus mamoratus et P. w. Triturus helveticus et $P$. w. Hynobius nebulosus et $P$. w.

\section{PLANCHE II}

Réactions de précipitation et d'immunoprécipitation sur gel d'agarose entre les produits de sécrétion de différents segments d'oviductes d'Urodèles.

$A, M, P, a, m, p$ : ont la mème signification que dans la Planche $I$.

$\sigma_{0}, \sigma_{1}, \sigma_{2}$ : désignent respectivement des sérums de lapins témoins $\left(\sigma_{0}\right)$ immunisés contre la gangue interne $\left(\sigma_{1}\right)$ ou la gangue movenne $\left(\sigma_{2}\right)$ de Pleurodèle.

$L_{1} I_{1}$ et $L_{2} I_{2}$ : désignent des arcs de précipitation (cf. fig. 2).

$\mathrm{L}_{1} \sigma_{1}, \mathrm{~L}_{2} \sigma_{1}$ et $\mathrm{L}_{1} \sigma_{2}$ : désignent des arcs d'immunoprécipitation.

Seuls les arcs reconnus de façon certaine ont été reproduits sur les schémas.

Noter la disparition apparente des arcs $L_{1} l_{1}$ en présence du sérum $\sigma_{2}$; en réalité, ces arcs sont déplacés vers les puits de dépôt de l'extrait renfermant $I_{1}$ en raison d'une communauté totale entre les arcs $L_{1} I_{1}$ et les arcs $I_{1}-\sigma_{2}$. Ceci peut être observé chez tous les Urodèles étudiés (le phénomène est cependant moins net chez Hynobius); il existe donc chez tous ces Urodèles un site antigénique commun reconnu par $L_{1}$. $L^{\prime}$ arc $\times$ n'est pas un arc d'immunoprécipitation, il correspond vraisemblablement à une réaction de précipitation. L'arc y provient de sites antigéniques communs à certains produits de A et de $M$. 
2. - Chez les autres Urodèles étudiés. Les extraits d'oviductes antérieur et moyen provenant d'Urodèles autres que Pleurodeles, forment en général plusieurs arcs d'immunoprécipitation lorsqu'ils sont confrontés aux sérums $\sigma_{1}$ ou $\sigma_{2}$ (anti-gangues ovulaires de Pleurodeles). Nous nous bornerons à discuter des arcs qui présentent des communautés avec les arcs $L_{1}-I_{1}$ ou $L_{2}-I_{2}$. On constate toujours l'absence apparente des arcs $L_{1}{ }^{-} l_{1}$ lorsque les extraits d'oviducte sont mis en présence de sérum $\sigma_{2}$; ce sérum produit d'ailleurs de manière constante au moins un arc d'immunoprécipitation contre les extraits d'oviductes moyens quelle que soit l'espèce considérée. Comme précédemment, Hynobius nebulosus semble se singulariser légèrement dans la mesure où il persiste, en présence du sérum $\sigma_{2}$ un arc ressemblant à ceux du groupe $L_{1}-I_{1}$ sans communauté avec les arcs d'immunoprécipitation de $I_{1}$ (qui sont pourtant très intenses avec cet animal)

3. - Chez les Anoures étudiés. - Chez Rana esculenta et Bufo bufo, les sérums 1 et 2 ne reconnaissent aucun antigène de façon nette. Chez Alytes obstetricans, le sérum $\sigma_{2}$ reconnaît un produit réparti à peu près également dans toutes les régions de l'oviducte.

\section{C. - Mise en évidence des réactions de précipitation dans les gangues ovulaires (Planche III).}

1. - Chez Pleurodeles walt/ (PI. III, A). - De très nombreuses expériences réalisées avec des gangues ovulaires déposées dans l'eau depuis plus de $12 \mathrm{~h}$, ont donné des résultats douteux ou franchement négatifs. En revanche, des extraits bruts prélevés dans les dix minutes qui suivent la ponte des ovocytes dans l'eau, sont capables de développer les différentes réactions de précipitation notamment lorsqu'ils sont confrontés aux extraits d'oviducte. Les ovocytes abondamment lavés ne forment aucun arc visible contre les extraits d'oviducte moyen (résultat non présenté); si on laisse autour des ovocytes la couche la plus interne de la gangue interne, on obtient une réaction de précipitation très nette avec les produits $I_{1}$ de l'oviducte moyen (PI. III, A). Juste après la ponte, les produits $L_{1}, L_{2}$ et $I_{1}$ sont présents dans les gangues ovulaires et sont capables de réagir pour former des arcs de précipitation.

2. - Chez d'autres Urodèles (PI. III, B). - Les expériences réalisées sur des gangues ovulaires prélevées tardivement, donnent des résultats négatifs tandis que celles réalisées sur des gangues ovulaires d'ovocytes fraîchement pondus (Hynobius) ou jeunes (Salamandra) sont capables de produire des réactions de précipitation (PI. III, B). Les communautés avec les arcs obtenus avec l'oviducte de Pleurodèle, indiquent que des produits de type $L_{1}$ et $I_{1}$ sont présents dans les gangues ovulaires de Salamandra et d'Hynobius.

Les résultats d'expériences réalisées avec les différents segments de l'oviducte d'un triton marbré femelle en train de pondre (ovocytes en transit), indiquent que les produits $L_{1}, I_{1}, I_{2}$ et très vraisemblablement $L_{2}$ sont présents dans les produits excrétés par l'oviducte de cet animal (résultats non présentés). 
(A)
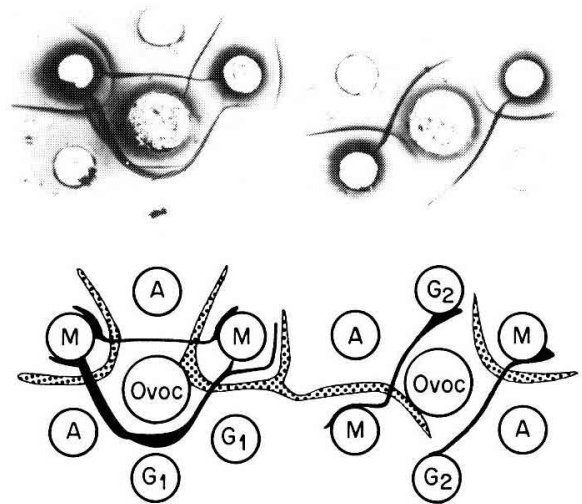

(B)

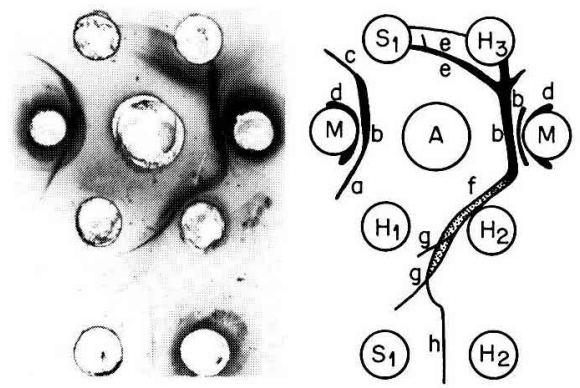

\section{PLANCHE III}

Réactions de précipitation obtenues avec les gangues ovulaires.

$A$ et $M$ : segments antérieur et moyen de l'oviducte du Pleurodèle (ces extraits ont été utilisés pour obtenir des arcs de référence).

\section{A. - Chez Pleurodèles.} tes.

$G_{1}$ et $G_{2}$ : gangues brutes prélevées sur des ovocytes déposés dans l'eau depuis quelques minu-

ovoc : extraits d'ovocytes correspondants, très sommairement débarrassés des gangues.

Les arcs de type $L_{1}-I_{1}$ sont représentés en pointillés; les arcs de type $L_{2}-l_{2}$ sont représentés en traits pleins.

Les gangues brutes renferment des produits $L_{1}, L_{2}$ et $I_{1}$ capables de réagir (en faibles quantités car ils ont déjà réagi entre eux en majorité). Les extraits bruts d'ovocytes renferment les produits $L_{1}$ et $I_{2}$ (les produits $L_{1}$ proviennent de gangue interne restée accolée à l'ovocyte puisque des extraits d'ovocytes lavés ou d'ovaire ne renferment pas ce produit (résultats non présentés)).

\section{B. - Chez Salamandra et Hynobius.}

$\mathrm{S}_{1}$ : gangue interne de Salamandra.

$\mathrm{H}_{1}$ : gangue interne d'Hynobius.

$\mathrm{H}_{2}, \mathrm{H}_{3}$ : gangues moyennes d'Hynobius.

En raison des communautés avec les arcs $b$, les arcs $a_{r}, c_{r}, e, f, g$ et $h$ sont des arcs de type $L_{1}-l_{1}$; l'arc $d$ est de type $L_{2}-L_{2}$. Ainsi !es gangues internes $S_{1}$ et $H_{1}$ renferment les produits $L_{1}$; les gangues moyennes $\mathrm{H}_{2}$ et $\mathrm{H}_{3}$ renferment les produits $\mathrm{I}_{1}$. 


\section{Discussion.}

Chez tous les Urodèles que nous avons étudiés, il existe, dans les régions antérieure et moyenne de l'oviducte, certaines molécules que nous avons appelées respectivement $L_{1}$ et $l_{1}$ qui possèdent la propriété de réagir entre elles pour former des précipitations visibles sous forme d'arcs après double diffusion en gel d'agarose. La possibilité de réaliser des réactions interspécifiques croisées, indique que les mécanismes moléculaires de ces réactions de précipitation et les sites réactionnels sont identiques ou apparentés chez tous les Urodèles étudiés. Ceci est confirmé, au moins pour l'une des deux catégories de molécules par les résultats de l'analyse immunochimique qui montrent que les sites réactionnels de $\mathrm{I}_{1}$ sont antigéniquement identiques ou apparentés chez tous les Urodèles étudiés.

En plus des molécules $L_{1}$, il existe dans l'oviducte antérieur de Pleurodeles walt/ des molécules $L_{2}$ capables de provoquer, elles aussi, des réactions de précipitation avec des molécules $I_{2}$ de l'oviducte moyen. Des molécules de type $L_{2}$ existent probablement chez Triturus marmoratus, Triturus helveticus, Salamandra salamandra et Hynobius nebulosus mais il n'a pas été observé de sites antigéniques communs aux différentes espèces pour ce type de molécules.

Dans les gangues ovulaires fraîchement excrétées, il est possible de mettre en évidence des produits $L_{1}, L_{2}$ et $I_{1}$ capables de réaliser des précipitations. En revanche, ceci est difficile voire impossible dans des gangues ovulaires excrétées depuis plusieurs heures à l'exception de composés de type $\mathrm{L}_{2}$ qui peuvent encore réagir avec de l'amidon (Jégo et al., 1983). Ceci tient très probablement au fait que les réactions de précipitation commencent à se produire spontanément dès que les produits sont excrétés par les cellules de l'oviducte ; plus le temps passe, moins il reste de produits $n^{\prime}$ ayant pas encore réagi. Cet aspect dynamique devra être approfondi mais il apparaît d'ores et déjà qu'au moment où les spermatozoïdes traversent les gangues ovulaires, dans les conditions naturelles, les produits $L_{1}$ et $L_{2}$ sont présents dans ces gangues et sont aptes à réaliser des réactions de précipitation. On peut, dans ces conditions, envisager l'intervention de telles molécules dans les interactions gangues ovulaires-spermatozoïdes chez les Urodèles. Leur absence dans les gangues ovulaires des Anoures, pourrait expliquer les différences entre ces derniers et les Urodèles dans les modalités de réalisation de la capacitation des spermatozoïdes et de la barrière à la polyspermie. On sait depuis longtemps capaciter artificiellement les spermatozoïdes d'Anoures (Aplington, 1957 ; Katagiri, 1966 ; Vorps et Elinson, 1979), alors que les expériences réalisées sur les spermatozoïdes d'Urodèles n'ont, à notre connaissance, jamais abouti à des capacitations. On peut imaginer dans ce deuxième cas, que seule l'intervention consécutive de différents facteurs, puisse déstabiliser la membrane du spermatozoïde et permettre à la réaction acrosomienne de se produire. Bien qu'il soit difficile de déterminer avec précision la région des gangues ovulaires où se produit la capacitation chez les Urodèles, il est raisonnable de situer ce phénomène dans la gangue moyenne ou à son voisinage immédiat. A l'entrée de la gangue moyenne, les spermatozoïdes $n$ 'ont subi aucune modification visible alors qu'ils ont perdu leur coiffe acrosomienne à la sortie de cette gangue (Piche- 
ral, 1977). Chez ces animaux, il est probable que la capacitation précède immédiatement la réaction acrosomienne et intervienne donc, soit juste avant la gangue moyenne (dans la partie interne de la gangue externe) soit, plus vraisemblablement, à l'intérieur de cette gangue moyenne. Des protéines basiques que nous avons mises en évidence dans la gangue moyenne (résultats non publiés) et qui n'existent pas dans les autres gangues, pourraient commencer à déstabiliser la membrane du spermatozoïde puis les molécules de type $L_{1}$ (celles qui $n^{\prime}$ ont pas encore réagi avec $I_{1}$ ) ou de type $L_{2}$ pourraient achever la déstructuration des membranes plasmique et acrosomienne permettant ainsi la libération des enzymes acrosomiennes.

Chez les Anoures, où la fécondation est monospermique, on peut envisager l'existence de deux mécanismes différents capables d'arrêter les spermatozoïdes surnuméraires : un blocage électrique (Cross et Elinson, 1980) et/ou un blocage chimique après expulsion du contenu des granules corticaux (Grey et al., 1976). Ces deux mécanismes sont mis en place juste après la pénétration du spermatozoïde fécondant et se déroulent tous les deux dans le voisinage immédiat de l'œuf, d'où la difficulté à déterminer lequel est physiologiquement efficace. Chez les Urodèles, les possibilités de blocage de spermatozoïdes au niveau des gangues ovulaires, sont tout à fait différentes. La fécondation interspécifique est possible (Kawamura, 1952; Twitty, 1964). La polyspermie est de règle et la pénétration du premier spermatozoïde dans l'ovocyte des Urodèles ne provoque pas de variations électriques membranaires comparables à celles observées chez les Anoures (Charbónneau, 1981), ni d'éclatement des granules corticaux qui n'existent apparemment pas chez ces animaux (Wartenberg et Schmidt, 1961). II existe cependant un mécanisme tardif de blocage des spermatozoïdes chez les Urodèles. Entre la gangue interne et la gangue moyenne, on constate en effet l'installation progressive d'une zone densifiée (Boisseau et al., 1974 ; Lostanlen et al., 1976). Vingt à trente minutes après la ponte des ovocytes (ou des œufs) dans l'eau, cette région séparant la gangue interne et la gangue moyenne devient complètement imperméable aux spermatozoïdes dont l'extrémité antérieure est immobilisée à ce niveau tandis que le flagelle continue ses mouvements. L'installation de cette barrière tardive a lieu qu'il y ait fécondation ou non. Nous pensons que la mise en place de cette zone densifiée arrêtant les spermatozoïdes, résulte des réactions $L_{1}{ }^{-} l_{1}$ qui se déroulent spontanément au sein des gangues ovulaires dès que celles-ci sont déposées autour des ovocytes. Ces réactions $L_{1}-I_{1}$ chez les Urodèles, peuvent alors être comparées aux réactions intervenant entre le contenu des granules corticaux (ovocytaires) et la gangue interne chez les Anoures (Grey et al., 1974, 1976 ; Wyrick ct al., 1974) dans la mesure où toutes les deux aboutissent à un blocage de spermatozoïdes. Bien sûr, l'intérêt physiologique des réactions $\left.L_{1}\right|_{1}$ observées chez les Urodèles apparaît secondaire (sauf peut-être pour les espèces ovovivipares), si on ne retient que l'efficacité du blocage à la polyspermie. II pourrait cependant s'agir d'un mécanisme de régulation biologique en cours d'installation; le particularisme apparent (qui devra être confirmé) d'Hynobius nebulosus pourrait être lié à la position systématique de cet animal qui peut être considéré comme le plus primitif des Urodèles étudiés. Chez les Anoures, des modifications dans les modalités 
du déroulement et surtout du déclenchement de ces réactions, les rendent très efficaces contre la polyspermie.

Recu en septembre 1982. Accepté en janvier 1983.

Remerciements. Nous remercions très vivement $M$. M. Boisseau, Picheral et Thorn pour les exemplaires d'animaux qu'ils nous ont donnés. Nous remercions très chaleureusement Mmes Bernard, Mathelier et Touzalin et M. M. Blanchet et Morille pour I'aide technique qu'ils nous ont apportée dans la réalisation de ce travail.

\section{Références}

APLINGTON H. W., 1957. The insemination of body cavity and oviducal eggs of Amphibia. Ohio J. Sci., 57, 91-99.

BATAILLON E., 1919. Analyse de l'activation par la technique des œufs nus et la polyspermie expérimentale chez les Batraciens. Ann. Sci. nat. Zool., 10, 1-38.

BOISSEAU C., JÉGO P., JOLY J., PICHERAL B., 1974. Organisation et caractérisation histochimique des gangues ovulaires sécrétées par l'oviducte de Pleurodeles waltlii Michah. (Amphibien, Urodèle, Salamandridé). C.R. Soc. Biol. (Paris), 168, 1102-1107.

CHARBONNEAU M., 1981. Etude des mécanismes membranaires contrôlant l'entrée des spermatozoïdes chez les Amphibiens Anoures et Urodèles. Th. $3^{\text {e }}$ Cycle, Univ. Rennes.

CROSS N. L., ELINSON R. P., 1980. A fast block to polyspermy in frogs mediated by changes in the membrane potential. Develop. Biol., 75, 187-198.

GOOD G. M., DANIEL J. F., 1943. Fertilization of coelomic eggs of Triturus torosus. Univ. Calif. Publ. Zool., 51, 149-158.

GREY R. D., WOLF D. P., HEDRICK J. L., 1974. Formation and structure of the fertilization envelope in Xenopus laevis. Develop. Biol., 36, 44-61.

GREY R. D., WORKING P. F., HEDRICK J. L., 1976. Evidence that the fertilization envelope blocks sperm entry in eggs of Xenopus laevis : interaction of sperm with isolated envelopes. Develop. Biol., 54, 52-60.

JÉGO P., 1974. Composition en glucides des différents segments de l'oviducte et des gangues ovulaires chez Pleurodeles walt/ Michah. (Amphibien, Urodèle). Comp. Biochem. Physiol., 48B, 435-446.

JÉGO P., 1976. Analyse des protides des gangues ovulaires de Pleurodeles walt/ii Michah. (Amphibien, Urodèle). Ann. Biol. anim. Bioch. Biophys., 16, 13-24.

JEGO P., ABALAIN J. H., WROBLEWSKI H., 1976. Réactions de précipitation de type " antigèneanticorps " entre produits de sécrétion de différentes régions de l'oviducte de Pleurodeles waltiil Michah. C.R. Acad. Sci. Paris, sér. D., 282, 767-770.

JÉGO P., CHESNEL A., LERIVRAY H., LE TALLEC H., 1983. Caractéristiques des réactions de précipitation entre les produits de sécrétion de l'oviducte du Pleurodèle ; identification d'une lectine. Reprod. Nutr. Dévelop., 23, 537-557.

KAMBARA J., 1953. Role of the jelly envelopes of toad eggs in fertilization. Ann. Zool. Jap., 26, 78-81.

KATAGIRI C., 1966. Fertilization of dejellied uterine toad eggs in various experimental conditions. Embryologica, 9, 159-169.

KAWAMURA T., 1952. Studies on hybridization in Amphibians. IV. Hybrids between Hynobius nebulosus (Schlegel) and Hynobius naevius (Schlegel). J. Sci. Hiroshima Univ., Ser. B-I, 13, 139-148.

LOSTANLEN D., BOISSEAU C., JOLY J., 1976. Données ultrastructurales et physiologiques sur l'utérus d'un amphibien ovovivipare Salamandra salamandra L., Ann. Sci. nat. Zool., $12^{e}$ s., 18, 113-114. 
METZ C. B., 1967. Gamete surface components and their role in fertilization, 163-236. In METZ C. B., MONROY A., Comparative morphology, biochemistry and immunology. Acad. Press, New York.

OUCHTERLONY O., 1949. Antigen-antibody reactions. Acta pathol. microbiol. scand., 26, 507-515.

PICHERAL B., 1977. La fécondation chez le triton pleurodèle. I. La traversée des enveloppes de l'œu par les spermatozoïdes. J. Ultrastr. Res., 60, 106-120.

SHAPIRO B. M., EDDY E. M., 1980. When sperm meets egg : biochemical mecanisms of gamete interaction, 257-302. In BOURNE G. H., DANIELLY J. F., Int. Rev. Cytol., vol. 66.

SHIVERS C. A., JAMES J. M., 1970. Capacitation of frog sperm. Nature, 227, 183-184.

TWITTY V. C., 1964. Fertility of Taricha species - hybrids and viability of their offspring. Proc. nat. Acad. Sci., USA, 51, 156-161.

VORPS M. M., ELINSON R. P., 1979. Extraction of oviducal materials important in the fertilization of Rana pipiens eggs. Biol. Reprod., 20, suppl. 1, 91A.

WARTENBERG H., SCHMIDT W., 1961. Elektronen mikroscopische Untersuchungen der strukturellen Veränderungen im Rindenbereich des Amphibieneies im Ovar und nach der Befruchtung. $Z$. Zellforsch. Mikroskop. Anat., 54, 118.

WYRICK R. E., NISHIHARA T., HEDRICK J. L., 1974. Agglutination of jelly coat granule components and the block to polyspermy in the Amphibian Xenopus laevis. Proc. nat. Acad. Sci., USA, 71, 2067-2071.

YUREWICZ E. C., OLIPHANT G., HEDRICK J. L., 1975. The macromolecular composition of Xenopus laevis egg jelly coat. Biochemistry, 14, 3101-3107. 